\title{
Active Hair Bundle Motion Linked to Fast Transducer Adaptation in Auditory Hair Cells
}

\author{
A. J. Ricci, ${ }^{1,2}$ A. C. Crawford, ${ }^{3}$ and R. Fettiplace ${ }^{1}$ \\ ${ }^{1}$ Department of Physiology, University of Wisconsin Medical School, Madison, Wisconsin 53706, ${ }^{2}$ Neuroscience Center, \\ Louisiana State University Medical Center, New Orleans, Louisiana 70112, and ${ }^{3}$ Department of Physiology, University of \\ Cambridge, Cambridge CB2 3EG, United Kingdom
}

\begin{abstract}
During transduction in auditory hair cells, hair bundle deflection opens mechanotransducer channels that subsequently reclose or adapt to maintained stimuli, a major component of the adaptation occurring on a submillisecond time scale. Using a photodiode imaging technique, we measured hair bundle motion in voltage-clamped turtle hair cells to search for a mechanical correlate of fast adaptation. Excitatory force steps imposed by a flexible glass fiber attached to the bundle caused an initial movement toward the kinocilium, followed by a fast recoil equivalent to bundle stiffening. The recoil had a time course identical to adaptation of the transducer current, and like adaptation, was most prominent for small stimuli, was slowed by reducing extracellular calcium, and varied with hair cell resonant frequency. In free-
\end{abstract}

standing hair bundles, depolarizations positive to $0 \mathrm{mV}$ evoked an outward current attributable to opening of transducer channels, which was accompanied by a sustained bundle deflection toward the kinocilium. Both processes were sensitive to external calcium concentration and were abolished by blocking the transducer channels with dihydrostreptomycin. The similarity in properties of fast adaptation and the associated bundle motion indicates the operation of a rapid calcium-sensitive force generator linked to the gating of the transducer channels. This force generator may permit stimulus amplification during transduction in auditory hair cells.

Key words: adaptation; hair cell; cochlea; hair bundle motility; mechanoelectrical transduction; photodiode imaging
Hair cells of the inner ear detect mechanical stimuli by deflections of the hair bundle, which gate mechanoelectrical transducer channels highly permeable to calcium (Corey and Hudspeth, 1979; Ohmori, 1985; Ricci and Fettiplace, 1998). Transduction is shaped by the kinetics of the transducer channels and the mechanical properties of the hair bundle, which have both passive and active components. In turtle auditory hair cells, bundles are comprised of 60-90 stereocilia (Hackney et al., 1993), whose passive compliance is dominated by the flexural rigidity of actin filaments in the narrowed stereociliary ankles (Crawford and Fettiplace, 1985). Bundle compliance is also determined by interstereociliary linkages, especially the tip-links, which have been suggested to transmit force to mechanotransducer channels (Pickles et al., 1984) thought to be located near the stereociliary tips (Hudspeth, 1982; Jaramillo and Hudspeth, 1992). Active or nonlinear contributions to hair bundle compliance normally assume reciprocal force transmission from the mechanotransducer channels to the hair bundle via the tip-links (Howard and Hudspeth, 1988).

The use of photodiode imaging techniques to monitor submicrometer motion of the hair bundle has revealed active bundle movements, manifested as spontaneous events or as reactions to stimuli such as force steps to the bundle or changes in hair cell membrane potential. Two categories of movement have been described with different time scales, most likely reflecting disparate mechanisms. One category is fast oscillations with a period of a few milliseconds (Crawford and Fettiplace, 1985; Howard and Hudspeth, 1987; Denk and Webb, 1992; Benser et al., 1996). The other

\footnotetext{
Received May 16, 2000; revised July 6, 2000; accepted July 12, 2000.

This work was supported by National Institutes on Deafness and other Communicative Disorders Grants RO1 DC 01362 (to R.F.) and RO1 DC 03896 (to A.J.R.) and a Deafness Research Foundation grant to A.J.R. We thank the reviewers for helpful suggestions on improving the Discussion.

Correspondence should be addressed to Robert Fettiplace, 185, Medical Sciences Building, 1300 University Avenue, Madison, WI 53706. E-mail: fettiplace@ physiology.wisc.edu.

Copyright (C) 2000 Society for Neuroscience $\quad 0270-6474 / 00 / 200001-12 \$ 15.00 / 0$
}

includes slower relaxations spanning tens or hundreds of milliseconds (Howard and Hudspeth, 1987; Rüsch and Thurm, 1990; Assad and Corey, 1992). A central hypothesis for the slower type of movement (Howard and Hudspeth, 1987; Assad and Corey, 1992) relates it to adaptation of the mechanotransducer channels, which resets the range of bundle movements detected by the channel during prolonged stimuli (Eatock et al., 1987; Crawford et al., 1989; Eatock, 2000). The slow resetting of sensitivity may involve a myosin-based motor that regulates tension in the tip-links (Hudspeth and Gillespie, 1994). The fast active component has been investigated less because of the difficulty of voltage clamping hair cells while monitoring bundle position with a rapid imaging technique.

By recording hair cell transducer currents in the turtle basilar papilla, we have recently distinguished two kinetic components of transducer adaptation (Wu et al., 1999). The main component observable for small bundle displacements has a time constant of 0.3-3 msec, is driven by calcium entry (Ricci and Fettiplace, 1998), and is insensitive to myosin ATPase inhibitors (Wu et al., 1999). Here we use photodiode imaging of the hair bundle to demonstrate rapid hair bundle movements coincident with the fast component of adaptation. These movements are opposite in polarity to those previously associated with adaptation (Howard and Hudspeth, 1987; Assad and Corey, 1992). We suggest that fast adaptation and the concomitant bundle movements are twin manifestations of a rapid calcium-feedback control of the mechanotransducer channels, which is separate from myosin adjustment of the tip-link attachment.

\section{MATERIALS AND METHODS}

Preparation and recording. Hair cells were whole-cell voltage clamped in the intact auditory papilla of the turtle (Trachemys scripta elegans) as described previously (Ricci and Fettiplace, 1997). Turtles (carapace length of $75-125 \mathrm{~mm}$ ) were decapitated, and a portion of the membranous labyrinth that included the cochlea was dissected out. The cochlea was opened and digested in saline [composition (in mM): $125 \mathrm{NaCl}, 4 \mathrm{KCl}, 2.8$ $\mathrm{CaCl}_{2}, 2.2 \mathrm{MgCl}_{2}, 2 \mathrm{Na}$ pyruvate, 8 glucose, and $\left.10 \mathrm{NaHEPES}, \mathrm{pH} 7.6\right]$ 
containing up to $0.06 \mathrm{mg} / \mathrm{ml}$ protease (type XXIV; Sigma, St. Louis, MO) The tectorial membrane was then lifted off to expose the hair bundles. Adequate enzymatic digestion before removal of the tectorial membrane was crucial for obtaining large mechanoelectrical transducer currents. The preparation was secured, hair bundles uppermost, with strands of dental floss in a Sylgard well of a lucite chamber. It was mounted on the stageof a Zeiss (Oberkochen, Germany) Axioskop FS microscope and viewed with a $63 \times$ water-immersion objective (numerical aperture of 0.9 ) and a Hamamatsu (Bridgewater, NJ) C2400 CCD camera. The recording chamber was perfused with saline of composition (in $\mathrm{mm}$ ): $128 \mathrm{NaCl}, 0.5 \mathrm{KCl}$, $2.8 \mathrm{CaCl}_{2}, 2.2 \mathrm{MgCl}_{2}, 2 \mathrm{Na}$ pyruvate, 8 glucose, and $10 \mathrm{NaHEPES}, \mathrm{pH}$ 7.6. The upper surface of the hair cell epithelium was separately perfused with a $\sim 100$ - $\mu$ m-diameter pipette introduced into the cochlear duct to control the solution bathing the hair bundle. This solution had a composition of (in $\mathrm{mm}$ ): $130 \mathrm{NaCl}, 0.5 \mathrm{KCl}, 2 \mathrm{Na}$ pyruvate, 8 glucose, and $10 \mathrm{NaHEPES}, \mathrm{pH}$ 7.6 , with free $\mathrm{Ca}^{2+}$ concentrations of $0.05,0.1,0.25$, or $2.8 \mathrm{~mm}$. The rate of perfusion and the distance of the perfusion pipette from the recording site were adjusted to ensure that the cochlear duct perfusion did not mechanically stimulate the bundle. In some experiments, $0.2 \mathrm{~mm}$ dihydrostreptomycin (DHS) (Sigma) was delivered in low-calcium saline to block the mechanotransducer channels.

Whole-cell currents were measured with an Axopatch 200A amplifier (Axon Instruments, Foster, City, CA) attached to a borosilicate patch electrode. Patch electrodes were filled with an internal solution containing (in mM): $125 \mathrm{CsCl}, 3 \mathrm{Na}_{2} \mathrm{ATP}, 2 \mathrm{MgCl}_{2}$, and $10 \mathrm{CsHEPES}$, adjusted to $\mathrm{pH}$ 7.2 with $\mathrm{CsOH}$. EGTA (1 mM; Fluka, Ronkonkoma, NY) was normally added to the internal solution as the calcium buffer, but in some experiments, 1 or $5 \mathrm{~mm}$ BAPTA (Molecular Probes, Eugene, OR) was used instead. After application of up to $70 \%$ series resistance compensation, electrode access resistances were 1-5 M $\Omega$, which gave recording time constants of $10-80 \mu \mathrm{sec}$. At the end of an experiment, the location of the hair cell on the long axis of the basilar papilla was noted. Peak mechanotransducer currents of 300-1200 pA were recorded at a holding potential of $-80 \mathrm{mV}$ in saline containing $2.8 \mathrm{~mm}$ calcium. Transducer currents and other experimental signals were stored on a Sony PCM instrumentation recorder at a band width of $0-10 \mathrm{kHz}$. Experiments were performed at $19-23^{\circ} \mathrm{C}$.
Hair bundle stimulation. Hair bundles were stimulated with a flexible glass fiber cemented to a piezoelectric bimorph (Crawford and Fettiplace, 1985). The free end of the fiber was placed in the rake of the bundle just above the level of the shortest stereociliary rank. The bimorph was driven differentially with voltage steps, shaped with an eight-pole Bessel filter at 2 $\mathrm{kHz}$, and amplified through a high-voltage driver of 20 -fold gain, to yield a $10-90 \%$ voltage rise time of $\sim 0.2 \mathrm{msec}$. Each flexible fiber was fabricated from a truncated patch pipette, the shank of which was drawn out to be $\sim 30 \mu \mathrm{m}$ long and less than $0.5 \mu \mathrm{m}$ in diameter. The mechanical stiffness of a fiber was calibrated by measuring with a horizontal microscope the deflection of the fiber produced by hanging polymethylmethacrylate beads on its tip (Howard and Ashmore, 1986). The weight of the spherical beads was calculated from their diameter assuming a polymethylmethacrylate density of $1190 \mathrm{~kg} / \mathrm{m}^{3}$. The stiffness of the fibers was between 0.4 and 1.4 $\mathrm{mN} / \mathrm{m}$. To minimize electrostatic repulsion between fiber and beads or between two fibers calibrated against each other, fibers were exposed to $\alpha$-radiation from a $\mathrm{Po}^{210}$ source (Staticmaster ionizer; Amstat Industries, Glenview, IL). The fiber was acid-cleaned at the start of each experiment to promote its adherence to the hair bundle membrane, thus ensuring that the bundle would follow faithfully movements of the fiber. The time course of the displacement of a fiber was determined by projecting its image onto a pair of photodiodes (Crawford and Fettiplace, 1985). Because short flexible fibers were used, thus minimizing their viscous drag, the $10-90 \%$ rise time of their motion in free solution was within $100 \mu \mathrm{sec}$ of the driving voltage. Previous failure to measure active bundle movements associated with transducer adaptation in turtle isolated hair cells (Crawford et al., 1989) may have been attributable to mechanical filtering imposed by the viscous drag on long $(>100 \mu \mathrm{m})$ stimulating fibers. In a few experiments, hair bundles were stimulated with a rigid glass probe fire polished to $1 \mu \mathrm{m}$ diameter at its tip as described previously (Ricci and Fettiplace, 1997).

Hair bundle imaging. The apparatus for measuring hair bundle motion was similar to that described previously (Crawford and Fettiplace, 1985; Crawford et al., 1989) with the sole technical difference that the hair bundle, rather than the attached fiber, was directly imaged on the photodiodes. An advantage of this approach was that the image of the bundle had much better contrast than the submicrometer diameter flexible fiber. It also enabled the unrestrained motion of the bundle to be observed (Denk
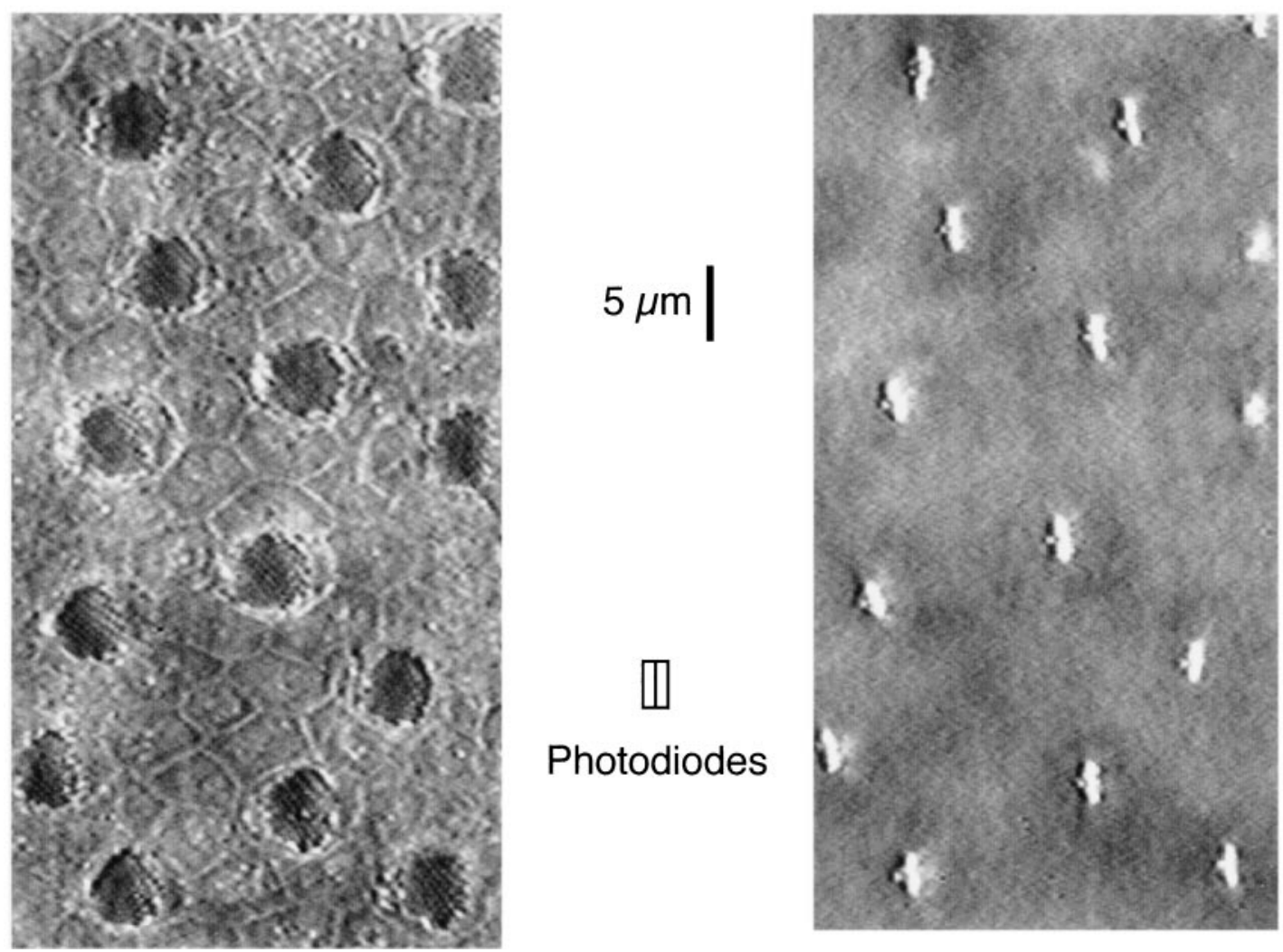

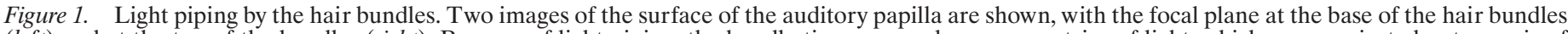

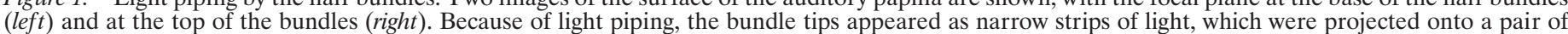

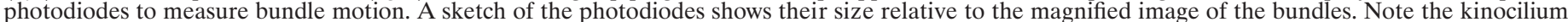
visible on the abneural aspect of each bundle. 
and Webb, 1990; Rüsch and Thurm, 1990). Under bright-field illumination from a $100 \mathrm{~W}$ tungsten-halogen lamp, the hair bundles behaved as light pipes and appeared as narrow bright lines when the microscope was focused at their tip (Fig. 1). To achieve such light piping, it was necessary to align the axis of the tallest stereocilia with the optical axis of the microscope. This was done by tilting the papilla, by introducing a glass coverslip under the neural limb and varying the tension in the strands of dental floss that secured the preparation. The secondary image of the bundle was projected onto a pair of photodiodes (Centronics LD 2-5) through the camera port of the Axioskop at a total magnification of $\sim 800 \times$. The photodiodes and current-to-voltage converter amplifiers were mounted on an $x-y$ translator that could be remotely positioned to center the bundle on the junction of the photodiodes. With $100 \mathrm{M} \Omega$ feedback resistors in the current-to-voltage converters, the differential photodiode signal was photon noise-limited and had a bandwidth of $2 \mathrm{kHz}$. In some experiments, the bandwidth was increased to $10 \mathrm{kHz}$ with frequency compensation amplifiers (Sigworth, 1983).

Provided excursions in the bundle image are less than the width of the photodiode pair, the difference between the signals from the two diodes should be proportional to the displacement of the image (Crawford and Fettiplace, 1985). The proportionality constant was determined for each hair bundle by scanning the diodes through a known distance across the image of the bundle and measuring the photocurrent (Art et al., 1986). The changes in photocurrent caused by bundle motion could then be calibrated because, from the magnification, $1 \mathrm{~nm}$ movement of the bundle in the object plane was equivalent to $800 \mathrm{~nm}$ of movement by the diodes in the image plane. For stimulating a hair bundle, the flexible glass fiber was attached to the sloping neural aspect of the bundle between 0.3 and 0.5 of the distance up the bundle from the epithelial surface. The vertical position of the fiber ensured that it was below the focal plane of the hair bundle tip, so that the light signal from the bundle was not contaminated by the image of the fiber. Assuming the hair bundles pivot about their base (Flock et al., 1977; Crawford and Fettiplace, 1985), motion of the attached end of the fiber will be $\lambda$ times the motion of the tip of the bundle, where $\lambda$ is the fractional distance of the fiber up the bundle $(\lambda=0.3-0.5)$. Furthermore, stiffness should decrease roughly as the square of the distance from the pivot (Crawford and Fettiplace, 1985; Howard and Ashmore, 1986). Therefore, if the photodetectors register a deflection, $x$, for the top of the hair bundle, the imposed displacement of the fixed end of a flexible fiber is $z$ and the stiffness of the fiber is $K_{F}$, the hair bundle stiffness, $K_{B}$, can be approximated by: $K_{B}=K_{F} \cdot \lambda^{2}(z-\lambda x) / \lambda x$.

\section{RESULTS}

\section{Hair bundle displacements with flexible fibers}

When the hair bundle was subjected to force steps with a flexible fiber, the transducer current behaved similarly to when displaced with a rigid probe. For forces toward the kinocilium, the current developed within a few tenths of a millisecond and then declined to a steady level, adaptation being fast and almost complete for the smallest stimuli. Such stimuli often produced hair bundle motion with a time course closely resembling that of the current (Fig. 2). Excitatory force steps with the flexible fiber evoked an initial displacement toward the kinocilium, followed by a fast recoil matching adaptation, that was equivalent to an increase in bundle stiffness. In some cells, after quickly attaining an initial peak, the hair bundle displacement decreased exponentially to a steady level (Fig. 2B); in other cells, the displacement declined from its peak to a secondary minimum and then increased again more slowly to a constant position (Fig. $2 A$; see Fig. $5 A$ ). At the end of the step, the bundle moved briefly past its steady-state position, reflecting an undershoot in the current.

The maximum amplitude of the initial displacement transient varied in different cells from 5 to $32 \mathrm{~nm}$. The reduction in displacement to a constant force step may be regarded as an increase in apparent stiffness of the bundle, although the underlying mechanism may be more complex than a simple change in the spring constants of the elastic elements of the bundle (see Discussion). For 14 cells, the chord stiffness was calculated (see Materials and Methods) as $1.2 \pm 0.6 \mathrm{mN} / \mathrm{m}$ (mean $\pm 1 \mathrm{SD}$ ) less than $1 \mathrm{msec}$ after the onset of the force step, and $2.2 \pm 1.6 \mathrm{mN} / \mathrm{m}$ in the steady state. Occasionally, there was a secondary minimum in the displacement response (Fig. $2 A)$. Such behavior is reminiscent of the under-damped oscillatory behavior observed previously in bundle motion (Crawford and Fettiplace, 1985) and in the voltage-clamped transducer current during
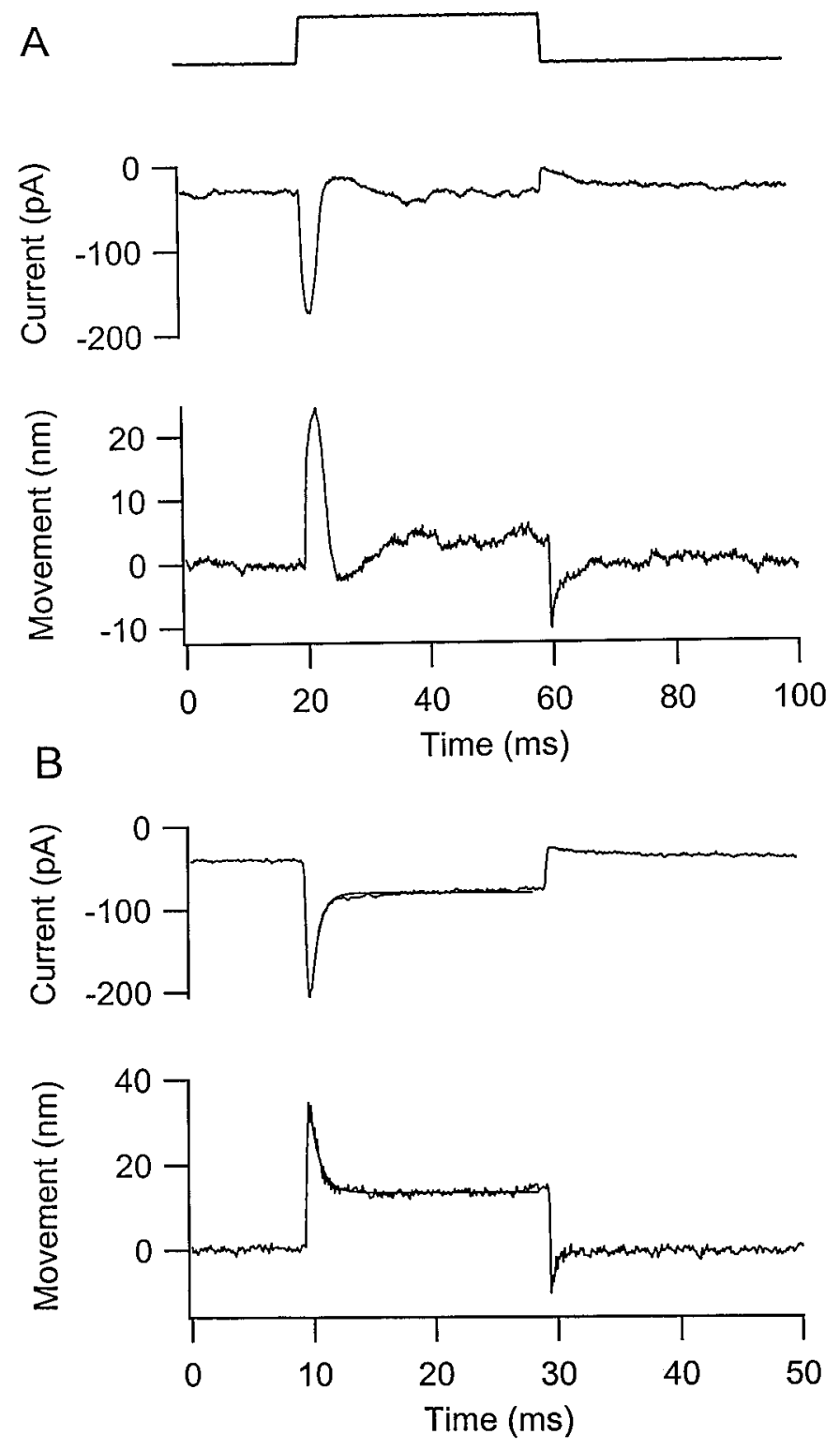

Figure 2. Active hair bundle motion elicited by force steps in two hair cells. In each cell, the hair bundle was stimulated with a flexible glass fiber producing a transducer current (middle) that resembled the bundle displacement (bottom). The time course of the deflection of the fixed end of the fiber, which approximates the timing of the force step, is shown at the top. In both examples, the stimulus magnitude was equivalent to a force of $37 \mathrm{pN}$ delivered to the tip of the bundle. In this and subsequent figures, positive displacements denote movement toward the kinocilium. The maximal transducer currents, recorded at $-80 \mathrm{mV}$, and the fractional distance of the cell along the basilar papilla from the low-frequency end whereas follows: $400 \mathrm{pA}, 0.36(A)$, and $800 \mathrm{pA}, 0.5(B)$. Superimposed on the records in $B$ are single-exponential fits to the current decay and the bundle recoil, with time constants of 0.7 and $0.8 \mathrm{msec}$, respectively. Each record is the average of 25 stimulus presentations. The intracellular calcium buffer was 1 mM EGTA.

stimulation with a rigid probe (Ricci et al., 1998). It implies that, under some circumstances, hair bundle mechanics are dominated by the gating properties of the mechanotransducer channels and, furthermore, can show frequency tuning.

In a given cell, the bundle recoil was most prominent for stimuli evoking less than half-maximal transducer currents. The magnitude of the initial displacement transient is plotted for three cells against the probability of opening of the mechanotransducer channels, calculated as the fraction of the transducer current activated 

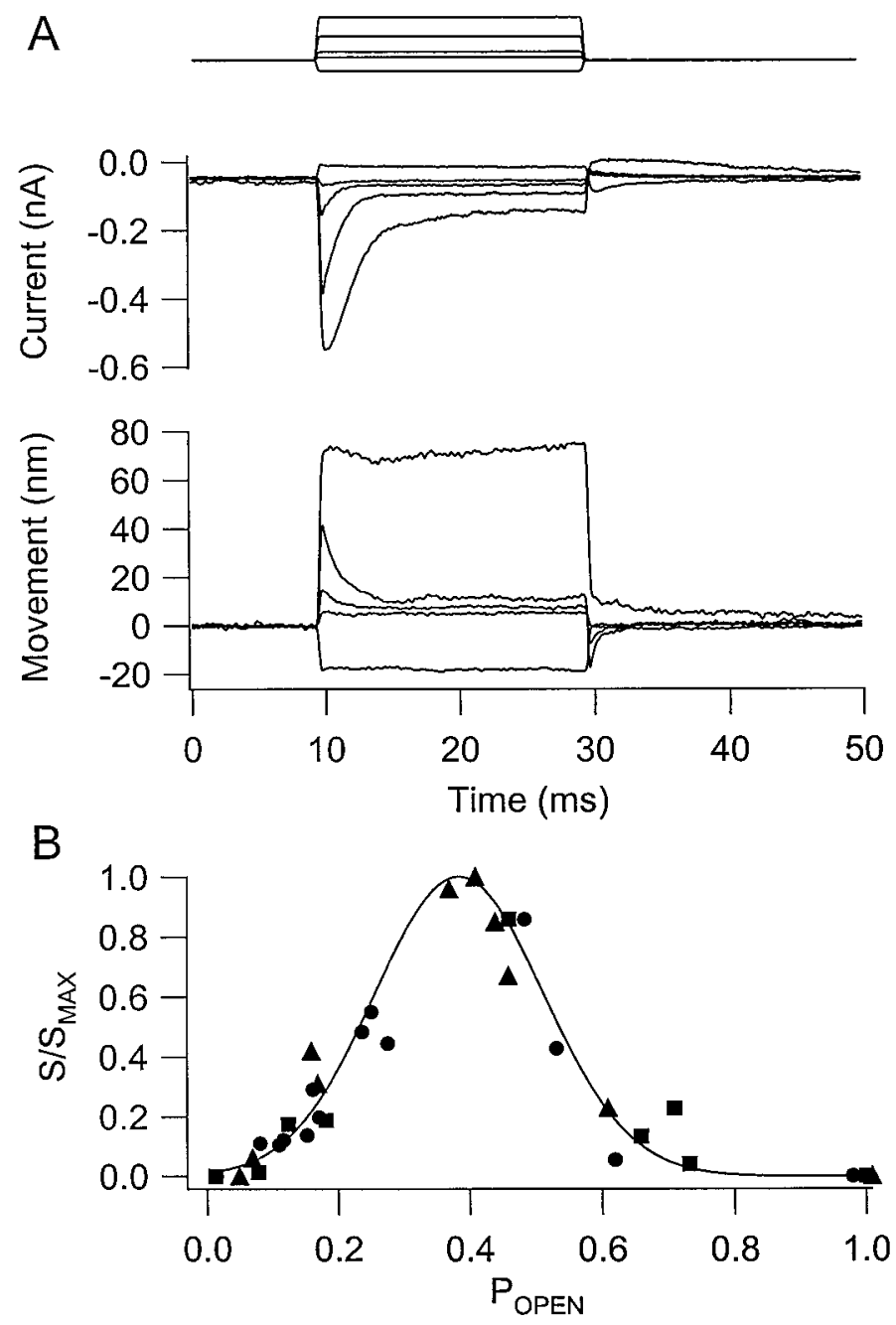

Figure 3. Dependence of the displacement transient on current magnitude. $A$, The hair bundle was stimulated with force steps (top), producing a family of transducer currents (middle) and associated bundle displacements (bottom). Note that the amplitude of the initial displacement transient, $S$, measured from the peak to the steady level, was largest for a current of intermediate size. Maximum transducer current (data not shown) was 800 $\mathrm{pA}$ at $-80 \mathrm{mV}$. Intracellular calcium buffer was $1 \mathrm{mM}$ EGTA. The largest force step was $31 \mathrm{pN}$. $B$, The displacement transient, $S$, scaled to its maximum value $S_{\mathrm{MAX}}$, is plotted against the probability of opening, $P_{\text {OPEN }}$, of the transducer channels for three different cells. $P_{\text {OPEN }}$ was calculated as the transducer current at the peak divided by its maximum value, $I_{\mathrm{MAX}}$, with a saturating stimulus. Values of $S_{\mathrm{MAX}}$ and $I_{\mathrm{MAX}}$ are as follows: squares, $32 \mathrm{~nm}, 840 \mathrm{pA}$; circles, $16 \mathrm{~nm}, 900 \mathrm{pA}$; triangles, $5.2 \mathrm{~nm}, 900 \mathrm{pA}$. Smooth curve is a gaussian: $S / S_{\mathrm{MAX}}=\exp \left[-\left(\left(P_{\mathrm{OPEN}}-0.39\right) / 0.18\right)^{2}\right]$

at the onset of the step (Fig. 3B). The results were fitted with a gaussian function that had a maximum when the probability of opening was $\sim 0.4$. With larger stimulus amplitudes, the initial transient comprised a smaller fraction of the overall bundle motion, despite the continued presence of an adaptive decline in the transducer current (Fig. 3A). As a consequence, the bundle movement and transducer current no longer possessed matching time courses. One manifestation of this difference was that the bundle displacement, after an initial transient, acquired a secondary growth in the same direction as the stimulus, despite a monotonic decline in the current. The delayed increase in displacement was sometimes seen even for the smallest stimulus amplitudes (Fig. $2 A$; see Fig. $5 A$ ), but in all cells, it was evident with larger stimuli. This type of motion resembles the slower component of bundle motion observed in bullfrog saccular hair cells (Howard and Hudspeth, 1987; Assad and Corey, 1992) and may signify the recruitment of another phase of adaptation.

\section{Speed of the displacement transient}

Inspection of the displacement and current records in Figure 2 indicates a close temporal correspondence between the two processes. A more quantitative comparison was obtained by superimposing scaled versions of the transducer current and bundle motion (Fig. $4 A$ ). During the falling phase of the response after the initial peak, the bundle motion lagged the current by at most $200 \mu \mathrm{sec}$, indicating that, within the limits of our measuring system, the two processes were effectively simultaneous. For most of the cells, the motion during the recoil was describable by a single exponential decay with a time constant similar to that of the decline in transducer current during fast adaptation. The time constants of both processes varied in different cells between $\sim 0.3$ and $2.5 \mathrm{msec}$ and were well correlated (Fig. 4B). However, most of the points on the correlation plot lie above the line of unity slope, indicating that the movement on average lagged the current by $0.2 \mathrm{msec}$. This could reflect a real delay between channel closure and bundle recoil but is more likely to represent a temporal limitation imposed by viscous drag on the bundle or the flexible fiber. Nevertheless, the similarity in the time courses of the mechanical and electrical events suggests that they are closely allied.

Much of the variation in the speed of adaptation is related to the location of the hair cell along the tonotopic axis of the basilar papilla. The resonant frequency of a hair cell increased systematically with its distance from the apical end of the papilla, an increase in resonant frequency being accompanied by a faster rate of adaptation (Ricci and Fettiplace, 1997; Ricci et al., 1998). Not surprisingly, therefore, the time constant of the bundle recoil was also correlated with hair cell position (Fig. 4C) and was faster in cells tuned to higher frequencies. The smooth curve through the experimental points in Figure $4 C$ was calculated from $\tau_{\text {mov }}=$ $5.7 \exp (-d / 0.37)$, where $\tau_{\text {mov }}$ is the time constant of the bundle relaxation and $d$ is the fractional distance of the hair cell along the papilla from the low-frequency apex. This same equation, derived from the tonotopic organization of the auditory papilla, was used to fit the change in adaptation time constant with hair cell position (Fettiplace and Fuchs, 1999) (Fig. 4).

\section{Covariation in fast adaptation and bundle motion}

The results so far suggest a causal relationship between fast adaptation and the initial displacement transient in the hair bundle motion. This conclusion was bolstered by experiments that varied adaptation in a given cell, demonstrating that the mechanical and electrical processes changed in parallel. Two types of experimental manipulation were used to slow the time course of adaptation: lowering extracellular $\mathrm{Ca}^{2+}$ concentration and reducing the holding potential (Fig. 5). In all cases, a change in the kinetics of adaptation was accompanied by an equivalent change in the hair bundle mechanical response.

Elevation of intracellular $\mathrm{Ca}^{2+}$ after its influx through open transducer channels is believed to be the primary signal driving adaptation. Consistent with this hypothesis, reducing the extracellular concentration of the divalent cation around the hair bundle from $2.8 \mathrm{~mm}$, its value in standard saline, to $50 \mu \mathrm{M}$ slows adaptation of the transducer current (Ricci and Fettiplace, 1998). In three experiments, lowering external $\mathrm{Ca}^{2+}$ produced equivalent increases in the time constants of fast adaptation and the decay of the displacement transient. In Figure $5 A$, the time constants of the electrical and mechanical responses were 0.4 and $0.6 \mathrm{msec}$, respectively, in $2.8 \mathrm{~mm} \mathrm{Ca}^{2+}$, which were increased to 1.4 and $1.3 \mathrm{msec}$, respectively, in $50 \mu \mathrm{M} \mathrm{Ca}{ }^{2+}$.

Changes in the holding potential also alter the rate of fast adaptation, probably by varying the driving force on $\mathrm{Ca}^{2+}$ entry through the transducer channels (Ricci and Fettiplace, 1998). Thus, hyperpolarization increases the driving force and accelerates adaptation. In three cells, varying the holding potential between 

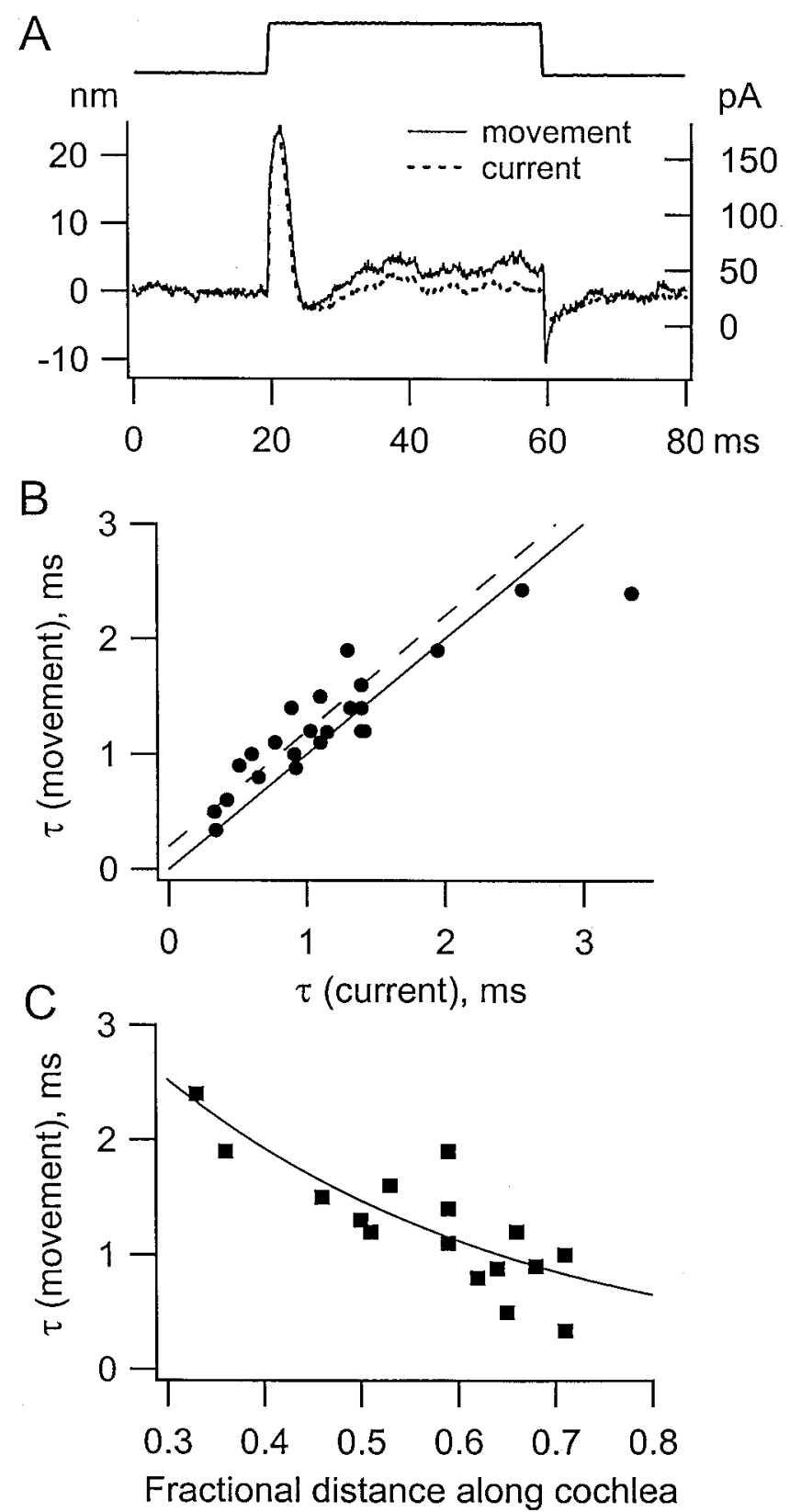

Figure 4. Time course of the displacement transient. A, Superposition of the transducer current and hair bundle displacement for the cell of Figure $2 A$. The relative sizes of the left-hand ordinate (bundle motion) and right-hand ordinate (transducer current) were adjusted to produce identical peak excursions of the two parameters. Note that, during the initial transient, the time courses of the current and bundle movement were almost identical, the movement lagging the current by no more than $0.3 \mathrm{msec}$. $B$, Collected results for 22 cells of the time constant of decay of the displacement transient plotted against the time constant of the transducer current adaptation. Values obtained from exponential fits as in Figure $2 B$. Note that the points lie above the continuous line of unity slope, suggesting a small delay between the movement and the current. Dashed line corresponds to the movement being delayed by $0.2 \mathrm{msec}$ with respect to the current. $C$, Time constant of decay of the displacement transient plotted against location of hair cell, expressed as the fractional distance, $d$, along the cochlea from the low-frequency end. All recordings made with $1 \mathrm{~mm}$ EGTA as intracellular calcium buffer. Hair cell resonant frequencies were estimated as $90 \mathrm{~Hz}$ at $d=0.3$ and $350 \mathrm{~Hz}$ at $d=0.8$. Smooth line was calculated from variation in the time constant of adaptation with cochlear location: $\tau($ movement $)=5.7 \exp (-d / 0.37)($ Fettiplace and Fuchs, 1999) .

-100 and $-20 \mathrm{mV}$ produced equivalent changes in the time courses of adaptation and of the displacement transient. Collected results of both holding potential and extracellular $\mathrm{Ca}^{2+}$ manipu-
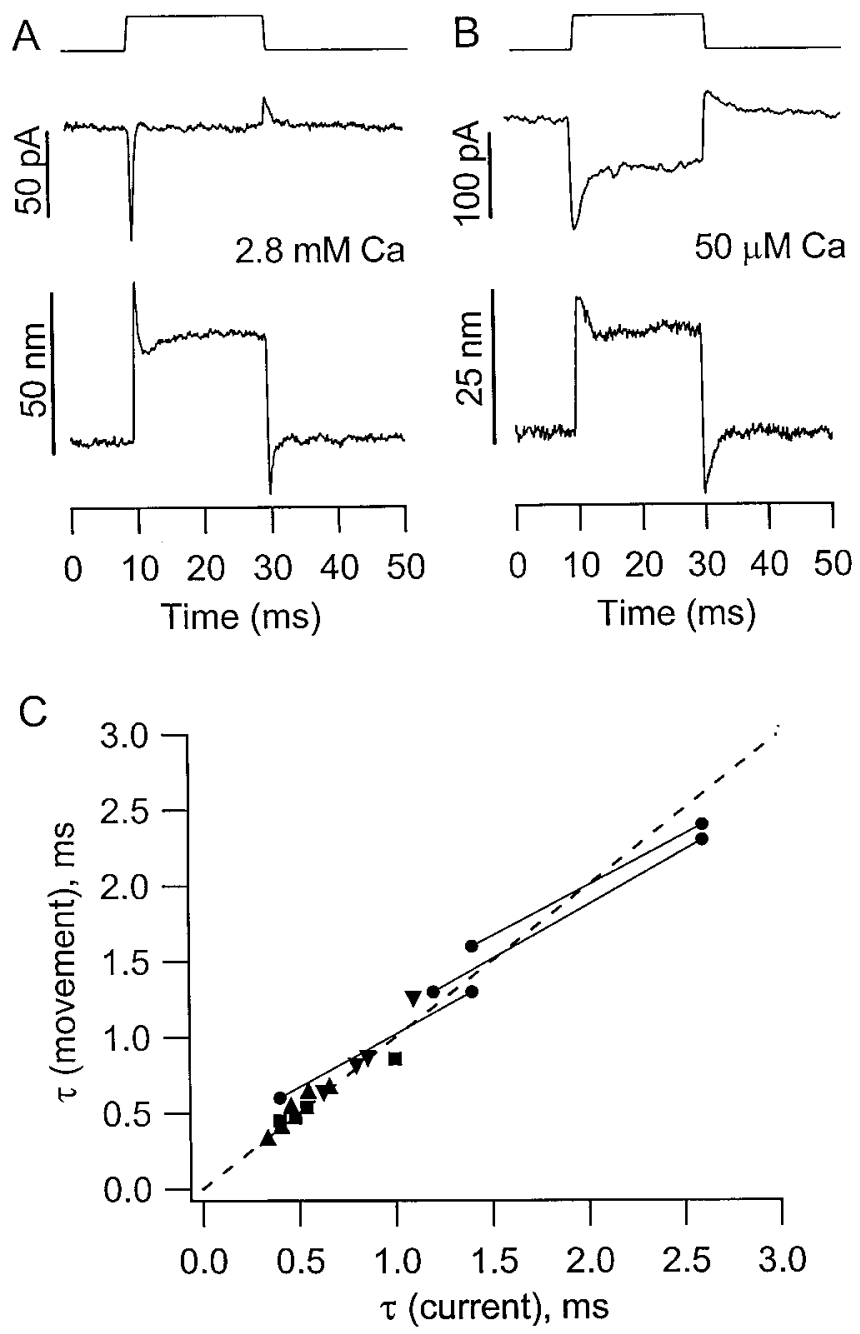

Figure 5. Covariation of the time constants of transducer adaptation and displacement transient with altered calcium and membrane potential. $A$, Transducer current and hair bundle displacement recorded in saline containing $2.8 \mathrm{~mm}$ calcium. $B$, Effects of perfusing saline containing $50 \mu \mathrm{M}$ calcium. Note that reducing the calcium slows the time course of the initial transient in both the current and displacement responses. Shown at the top is the timing of a force step equivalent to $85 \mathrm{pN}$ delivered to the tip of the bundle. EGTA (1 mM) was used as intracellular calcium buffer. Each record is the average of 25 responses. $C$, Correlation between time constant of displacement transient, $\tau$ (movement), and time constant of adaptation, $\tau$ (current), for changes in extracellular calcium (circles) and in holding potential (squares, triangles, and inverted triangles). Each pair of circles joined by a straight line are measurements on hair cells at $-80 \mathrm{mV}$ in $2.8 \mathrm{mM}$ and $50 \mu \mathrm{M}$ calcium as in $A$, low calcium increasing both the electrical and mechanical time constants. In three cells, time constants were measured in $2.8 \mathrm{~mm}$ at potentials between -100 and $-20 \mathrm{mV}$. The dashed line has a unity slope.

lations are shown in Figure $5 B$, confirming the correlation between the two processes. The correlation in kinetics confirms the relationship between the fast adaptation of the transducer current and the bundle displacement response to a force step.

\section{Hair bundle deflections generated by depolarization}

Hair bundle motion could also be induced by changes in membrane potential that affect the gating of the transducer channels indirectly through altering intracellular calcium. Depolarization from -80 to $+80 \mathrm{mV}$ activated a large sustained outward current that developed over the course of a few milliseconds. On repolarization, the current became large and inward but quickly declined to a steady value. Most of the current induced by depolarization probably flowed through the mechanotransducer channels. It could be sup- 
A
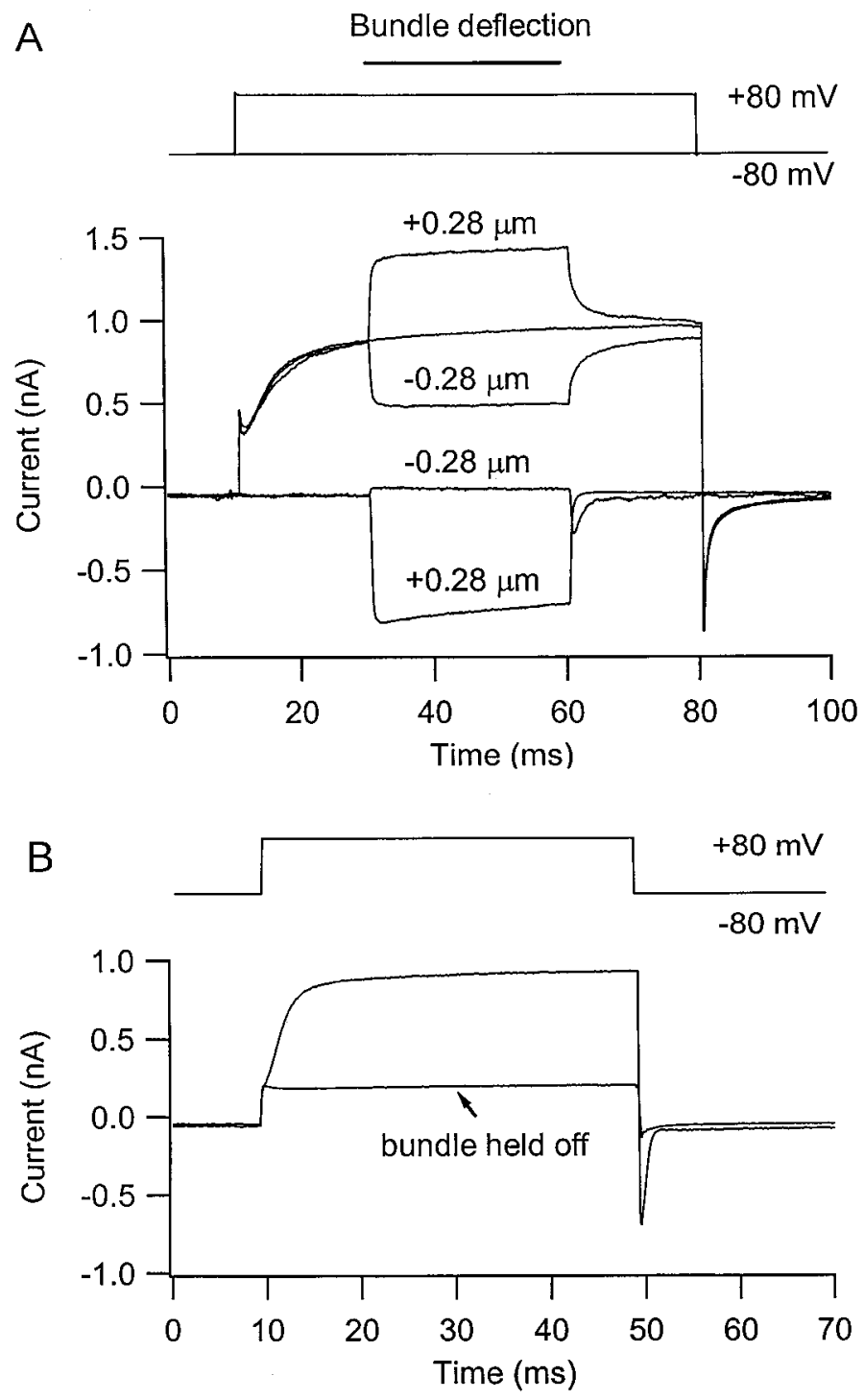

Figure 6. Transducer current activated by depolarization. $A$, Superimposed traces of transducer currents for $\pm 0.28 \mu \mathrm{m}$ step deflections of the hair bundle produced by a rigid glass probe. The currents are markedly asymmetric for positive and negative steps at $-80 \mathrm{mV}$ but become more symmetric during depolarization to $+80 \mathrm{mV}$. At the onset of the depolarization, there is slow activation of an outward current attributed to an increase in transducer current. Time course of bundle deflection and depolarization are shown at top. B, Activation of the outward current by depolarization can be prevented by maintained displacement of the hair bundle away from the kinocilium (bundle held off), indicating that it is attributable to the opening of transducer channels. Records in both $A$ and $B$ are averages of 25 presentations.

pressed by strong negative deflections of the hair bundle (Fig. 6B) and, moreover, was associated with an increase in the fraction of transducer current turned on at rest. In Figure $6 A$, this fraction changed from $\sim 5 \%$ at $-80 \mathrm{mV}$ to $50 \%+80 \mathrm{mV}$.

The magnitude of the time-dependent outward current was also correlated with the peak transducer current. The size of the current at $+80 \mathrm{mV}$ varied in different cells between 0.2 and $1 \mathrm{nA}$, which when expressed as a proportion of the transducer current had a value of $0.72 \pm 0.26$ (mean $\pm 1 \mathrm{SD} ; n=23 ; 1 \mathrm{~mm}$ EGTA). Consistent with earlier observations (Assad et al., 1989; Crawford et al., 1989; Ricci and Fettiplace, 1998), the outward current is likely to be activated by a drop in intracellular calcium. The fraction of mechanotransducer channels open at the resting position of the bundle is thought to be regulated by the calcium concentration at the inner face of the channel, determined by the balance between entry of the calcium and its subsequent buffering and extrusion. Therefore, depolarization toward the calcium equilibrium potential, which reduces calcium influx, will lower intracellular calcium concentration and open the mechanotransducer channels.

Depolarizing voltage steps to $+80 \mathrm{mV}$ also induced a sustained displacement of the hair bundle toward the kinocilium, occurring contemporaneously with the outward current. This movement was of the opposite polarity to that reported previously during depolarization of saccular hair cells (Assad and Corey, 1992). Many cells also showed a negative transient before the larger positive movement. On repolarization to $-80 \mathrm{mV}$, the hair bundle returned rapidly to its resting position, with a time constant matching the current relaxation that could be as brief as $0.3 \mathrm{msec}$. The most complete data on the voltage-induced movements were obtained in free-standing bundles in which the peak displacement varied in different cells between 10 and $80 \mathrm{~nm}$ and was directly proportional to the amount of outward current activated (Fig. $7 B$ ). Thus, larger movements were accompanied by the opening of more transducer channels. In one cell, a normal voltage-induced displacement was observed in a hair bundle with a detached kinocilium, suggesting that the movement is not a property of the kinocilium, which can itself be motile (Rüsch and Thurm, 1990). These observations argue that the bundle motion is associated with the gating of the mechanotransducer channels in the stereocilia.

\section{Time course of voltage-induced movement}

The hair bundle displacement occurred simultaneously with the development of the outward current. Superposition of scaled versions of the current and the movement (Fig. $8 \mathrm{~A}$ ) gave no evidence of the hair bundle motion either preceding or lagging the current by $>0.2$ msec. In some cells (Fig. 8 ), the hair bundle moved to its new position with a single time constant of a few milliseconds (mean of $5.4 \pm 2.3 \mathrm{msec} ; n=7$ ) at the onset of the depolarization and under $1 \mathrm{msec}$ at the offset. In other cells (Fig. $7 A$; see Fig. 10 $A$ ), there was a second slower time constant at both the onset and offset of the voltage step (mean of $15.6 \pm 1.5 \mathrm{msec} ; n=17$ ). In such cases, the time constant of the movement and current were highly correlated. Figure $8 B$ shows that the major (fast) time constants of the movement and current at the onset of the depolarization were almost identical.

The time course of events occurring at the onset of the depolarization, after suppression of calcium influx, are likely to be dominated by calcium diffusion away from the channel and extrusion from the stereocilium. At the end of the voltage step, calcium flows in through transducer channels opened during the depolarization, and the inward tail current declines as the channels close in a process resembling transducer adaptation. Further evidence of a link between hair bundle motion and fast adaptation was obtained by comparing the speed of the return of the bundle at the end of the depolarizing step with the kinetics of adaptation (Fig. $8 C$ ). For each cell, the adaptation time constant was measured from the responses to small bundle deflections produced by a rigid probe. In 24 cells recorded with $1 \mathrm{~mm}$ EGTA as the internal calcium buffer, fast adaptation had a time constant of $0.92 \pm 0.09$ msec (mean \pm 1 SEM), and the mechanical relaxation had a time constant of $0.82 \pm$ 0.07 msec. There was a good correlation between the time constants of the electrical and mechanical parameters for each cell (Fig. 8C).

If hair bundle motion reflects the process of fast adaptation, it should, like adaptation, be susceptible to the extracellular calcium concentration. Reducing external calcium from 2.8 to $0.25 \mathrm{~mm}$ produced a slowing of both the tail current and the bundle relaxation at the end of the depolarizing voltage step (Fig. 9). Measurements on five cells gave a time constant for the mechanical relaxation of $1.2 \pm 0.2 \mathrm{msec}$ (mean $\pm 1 \mathrm{SEM}$ ) in $2.8 \mathrm{~mm}$ calcium and $11.2 \pm 4.0 \mathrm{msec}$ in $0.25 \mathrm{~mm}$ calcium. An explanation for this behavior is that, on repolarization, less calcium enters the hair bundle in the lower calcium concentration, and the rate of closing of the transducer channels and of the attendant mechanical events are both slowed. Similar calcium effects on the rate of adaptation to 


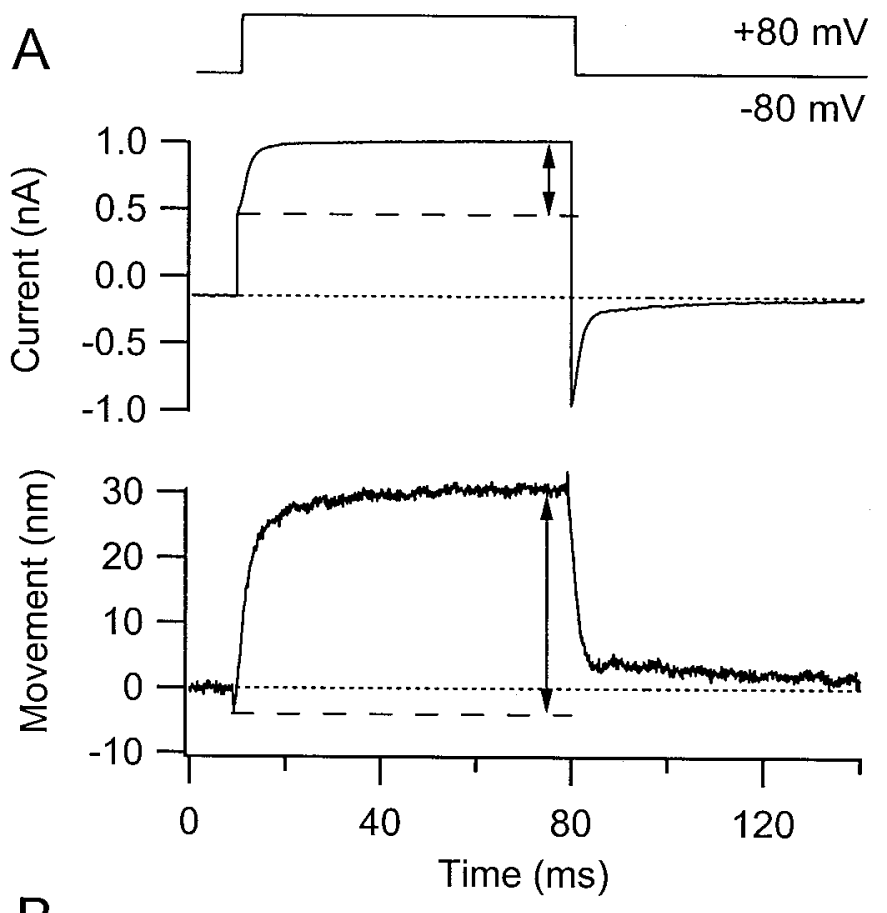

B

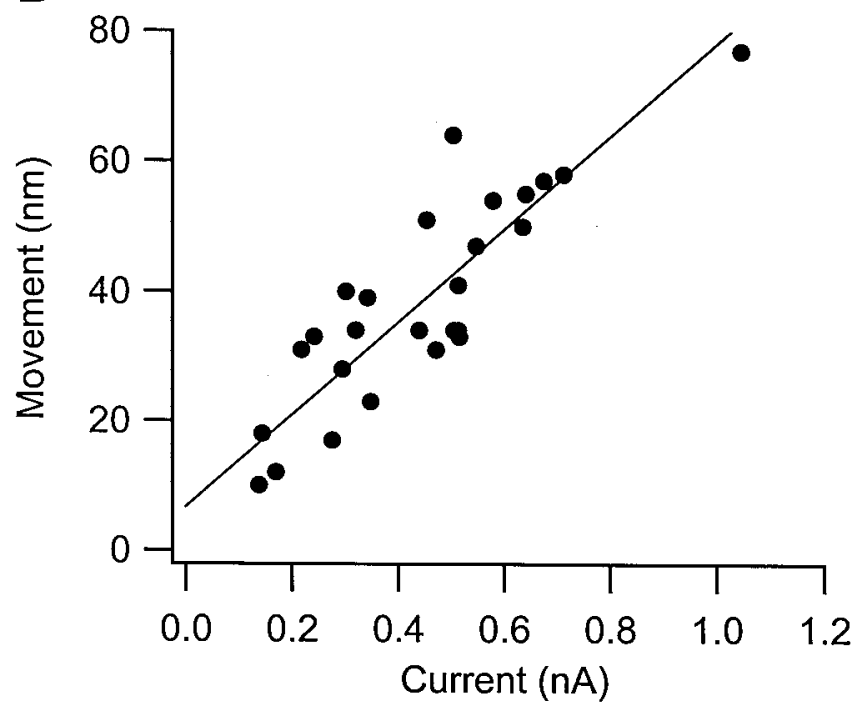

Figure 7. Movement of a free-standing hair bundle caused by depolarization. $A$, At the onset of the depolarizing step from -80 to $+80 \mathrm{mV}$, there was a transient movement away from the kinocilium, followed by a larger movement toward the kinocilium (bottom trace), with a time course similar to the development of the outward current (middle trace). Both traces are averages of 25 responses. $B$, Correlation between the magnitude of the hair bundle displacement during depolarization to $+80 \mathrm{mV}$ and the size of the outward current in 26 cells with $1 \mathrm{~mm}$ EGTA as intracellular calcium buffer. The bundle movement and current were measured as shown by the arrows in A. Straight line is least-squares fit with correlation coefficient of 0.89 .

hair bundle displacement have been observed previously (Ricci and Fettiplace, 1998). A second consequence of reduced external calcium was an increase in the holding current attributable to a larger fraction of transducer channels being open at the resting position of the bundle. In $2.8 \mathrm{~mm}$ calcium, only a small fraction of channels were open at rest, so hyperpolarization had little effect on either the electrical or mechanical manifestations of adaptation (Fig. 9A). In $0.25 \mathrm{~mm}$ calcium, the increased calcium influx with hyperpolarization had a larger number of open channels to exert its effect on and to close. At the onset of the hyperpolarizing voltage step, there was a surge of inward (transducer) current, exceeding $2 \mathrm{nA}$ in Figure 9B, which then declined because of adaptation. Accompanying this adaptive closure of channels was a movement of the hair bundle away from the kinocilium. Consequently, in 0.25 mM calcium, positive and negative voltage steps of equal amplitude evoked more nearly symmetric mechanical responses than in 2.8 $\mathrm{mm}$ calcium. However, the deviation in hair bundle position summed over the two voltage steps $(\sim 45 \mathrm{~nm})$ was identical in high and low calcium, reflecting a shift in the transducer activation range. Although lowering the calcium always led to an increase in the amount of transducer current turned on at rest (Fig. 9), which is consistent with a movement of the resting position of the bundle toward the kinocilium, slow drifts in the image of the bundle prevented confirmation of this point.

\section{Two components of hair bundle movement}

The analysis so far has focused on the large sustained hair bundle displacements that for depolarization are directed toward the kinocilium. At the onset of a depolarizing step, there was usually a smaller transient deflection away from the kinocilium preceding the slower sustained motion toward the kinocilium. Both components of bundle movement were graded with the size of the voltage step (Fig. 10). In all cells studied, the larger slower component varied nonlinearly with membrane potential and became prominent only for depolarizations positive to $0 \mathrm{mV}$. In contrast, the size of the fast component was proportional to the voltage change. From arguments presented so far, the major component of bundle motion most likely reflects changes in the probability of opening of the mechanotransducer channels secondary to variations in intracellular calcium. Its nonlinear voltage sensitivity may therefore be a combined result of the dependence of calcium entry on membrane potential and interaction of calcium with an intracellular saturable binding site.

The two opposing components of bundle motion could be separated by use of blockers of the transducer channel, such as DHS. Treatment with $0.2 \mathrm{~mm}$ DHS in low $(0.1 \mathrm{~mm})$ external calcium abolished the major displacement, as well as the outward current evoked by depolarization (Fig. 11). In the presence of the blocking agent, there remained an approximately rectangular response equivalent to a sustained movement away from the kinocilium. Both the time course of the response (Fig. 11B) and linear dependence on membrane potential (Fig. $10 B$ ) suggest it is driven by voltage but is not primarily related to gating of the mechanotransducer channels. In summary, depolarization produces two components in the photodiode signal from the hair bundle. The main component is a displacement toward the kinocilium, reflecting the opening of transducer channels; the other, of unknown origin, is equivalent to a smaller displacement away from the kinocilium, reflecting the time course and amplitude of the driving voltage.

\section{DISCUSSION}

\section{Active hair bundle motion related to transducer channel gating}

There is abundant evidence that mechanoelectrical transduction in hair cells can operate in both forward and reverse directions as proposed by Weiss (1982). In the forward direction, hair bundle motion generates electrical signals through the gating of the mechanotransducer channels. Reverse transduction involves active bundle motion driven by changes in hair cell membrane potential or additional force production by the bundle during stimulation with a compliant probe. Here, we have obtained evidence in turtle auditory hair cells linking fast reverse transduction to the state of the mechanotransducer channels. The polarity of reverse transduction was such that when the transducer channels opened, as with large depolarizations, the bundle moved toward the kinocilium, and when the channels closed, during adaptation or with hyperpolarization, there was a movement away from the kinocilium. Adap- 

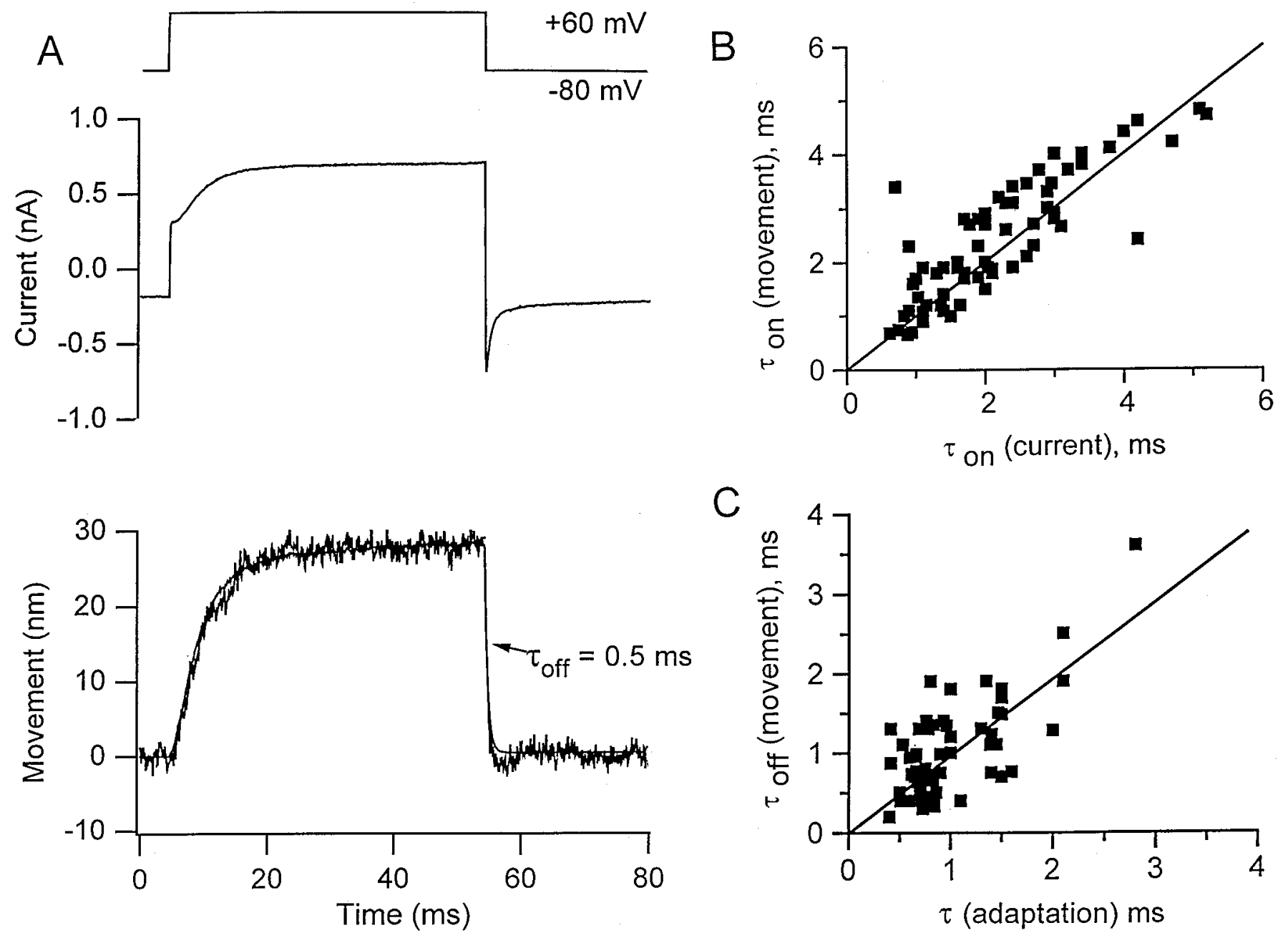

Figure 8. Speed of bundle movements related to opening and closing of transducer channels. $A$, Bundle displacement and current produced by depolarization from -80 to $+60 \mathrm{mV}$. A scaled version of the time-dependent component of the current activated during the depolarizing step has been superimposed on the bundle displacement record. This current is attributed to opening of transducer channels, which occurs with a time course identical to that of the hair bundle movement. $B$, Correlation between time constant of bundle movement and current. The principle time constant, $\tau_{\text {on }}$ (movement), of the displacement at the onset of the depolarization is plotted against the time constant, $\tau_{\text {on }}$ (current), with which the current develops. Line is least-squares fit through the origin with slope 1.05 and correlation coefficient 0.83 . $C$, Correlation between time constant of bundle movement and adaptation. The time constant, $\tau_{\text {off }}$ (movement), of bundle movement at the end of the depolarizing step is plotted against the time constant, $\tau$ (adaptation), of the adaptive decline in current during a small bundle displacement. In both $B$ and $C$, results are pooled from recordings with different intracellular calcium buffers, $1 \mathrm{~mm}$ EGTA, $1 \mathrm{~mm}$ BAPTA, and $5 \mathrm{~mm}$ BAPTA. Line is fit through the origin with slope 0.995 and correlation coefficient 0.70.

tive changes in channel gating and active hair bundle motion occurred with time constants as brief as $0.3 \mathrm{msec}$ and, within the limits of the measuring system, were simultaneous (Figs. 4, 8). Moreover, both processes were comparably sensitive to variations in extracellular calcium (Figs. 5, 9), suggesting that fast adaptation and the concomitant bundle movements are twin manifestations of a rapid calcium-feedback control of the transducer channels. Channels opened during extrinsic bundle deflection permit influx of calcium that closes them with attendant force production to promote adaptation.

If calcium binds either directly to the channels or to an accessory protein, the rate of adaptation may be limited by the gating kinetics of the channels themselves. The transducer current in turtle hair cells activates at room temperature with a time constant of 0.2-0.4 msec (Crawford et al., 1989), similar to the fastest time constant found for adaptation. However, in the mammalian cochlea, the hair cell transducer channels must be gated cyclically at frequencies up to tens of kilohertz. Therefore, in the mammalian auditory organ, active bundle motion of the type reported here could conceivably occur on a sufficiently fast time scale to supply energy on a cycle- by-cycle basis to the vibrations of cochlear partition and amplify low-level stimuli as in lower vertebrate hair cells (Martin and Hudspeth, 1999).

\section{Comparison with previous observations of active bundle movements}

The active hair bundle movements reported here are probably of identical origin to the oscillations in bundle position seen previously in turtle hair cells (Crawford and Fettiplace, 1985). The earlier measurements were made under current clamp and so were accompanied by large voltage oscillations that were manifestations of electrical tuning. Oscillatory bundle movements would therefore arise in part from changes in membrane potential and in part from calcium-feedback control of the transducer channels, which itself can produce oscillatory transducer currents (Ricci et al., 1998). Calcium feedback on to the channels might be expected sometimes to generate mechanical oscillations of the hair bundle even under voltage-clamp conditions. Figure $2 A$ is an example, although displacement responses with more oscillations might be obtainable 

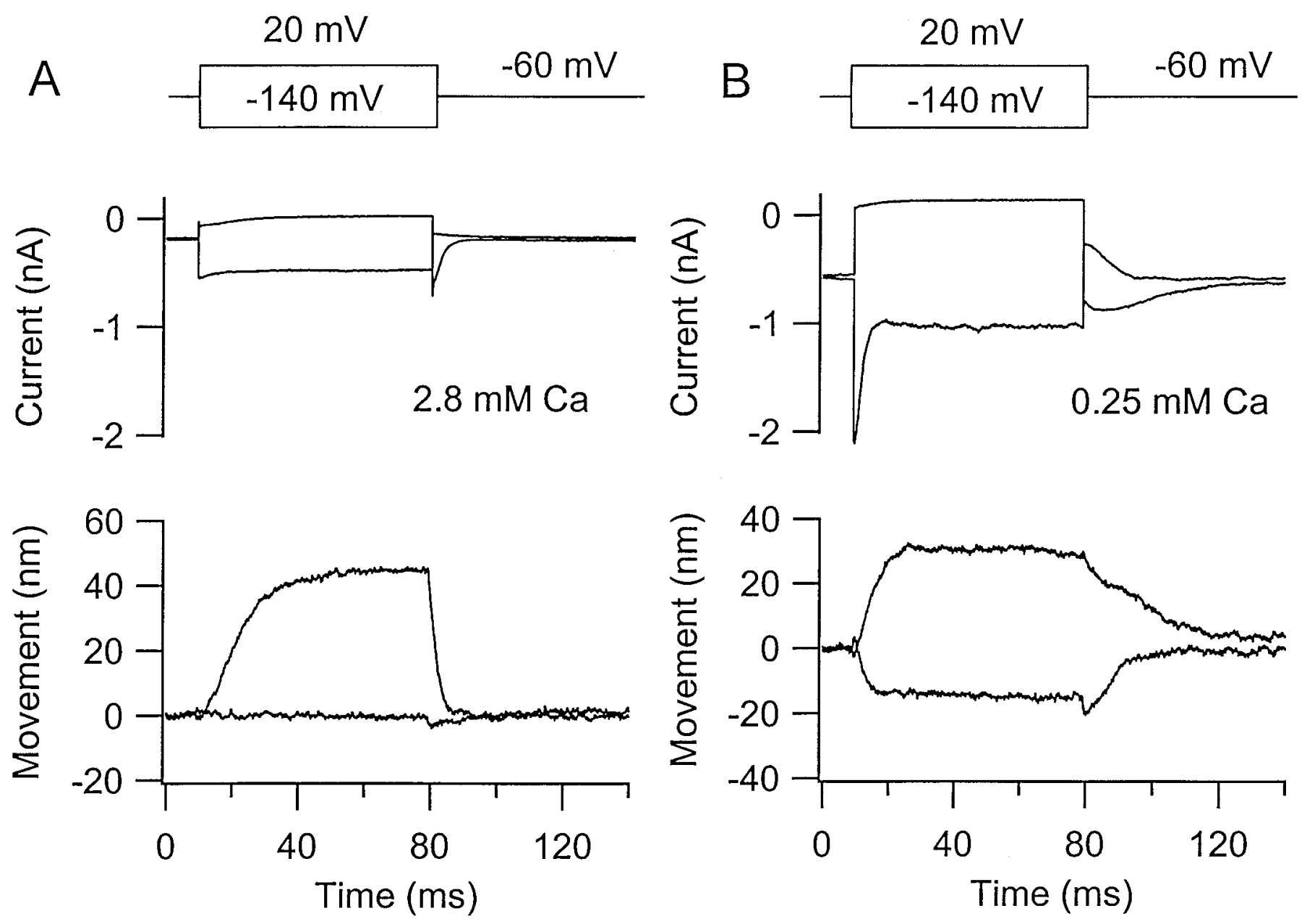

Figure 9. Effects of extracellular calcium on depolarization-induced movements. Average membrane currents and hair bundle movements during \pm 80 $\mathrm{mV}$ steps from $-60 \mathrm{mV}$ in $2.8 \mathrm{~mm}$ calcium $(A)$ and $0.25 \mathrm{~mm}$ calcium $(B)$. Both current relaxation and bundle movement on repolarizing to $-60 \mathrm{mV}$ slowed with the decrease in calcium concentration. The relative movements for the +80 and $-80 \mathrm{mV}$ steps differed in the two calcium solutions, but the total bundle excursion $(45 \mathrm{~nm})$ was similar. This may reflect $\sim 20 \mathrm{~nm}$ displacement of the bundle of toward the kinocilium in $0.25 \mathrm{~mm}$ calcium. Twenty-five responses were averaged in each condition.

given the higher quality factors of resonance observed with the transducer current (Ricci et al., 1998). The bundle movements also strongly resemble the rapid component of bundle motion observed in frog saccular hair cells, which has been referred to as a "notch" (Howard and Hudspeth, 1987) in the displacement record or a "twitch" (Benser et al., 1996). Our experiments suggest that the mechanical events are not inherently transient, but their time course reflects the decay of the transducer current. Indeed, depolarizing voltage steps evoked sustained hair bundle displacements that are a manifestation of the same active process.

Slower components of active bundle motion, on the time scale of tens of milliseconds, have also been described in frog hair cells. In response to a force step, these appeared as a slow growth of bundle displacement to a steady state. Such mechanical relaxations had average time constants of $27 \mathrm{msec}$ in response to flexible-fiber stimulation (Howard and Hudspeth, 1987) and $59 \mathrm{msec}$ at the end of a depolarizing voltage step (Assad and Corey, 1992). The polarity of these movements was the opposite of those observed here. With a paradigm identical to ours, depolarizations positive to $0 \mathrm{mV}$ elicited an outward current because of opening of mechanotransducer channels, which was accompanied by hair bundle displacement away from the kinocilium. The difference in kinetics and polarity imply a separate mechanism postulated to underlie transducer adaptation, which in frog saccular hair cells often has a comparably slow time course (Assad and Corey, 1992). Transducer currents in turtle auditory hair cells are dominated by rapid adaptation, especially for small bundle displacement around rest. However, a slower component of adaptation can be recruited at larger bundle deflections (Wu et al., 1999). Additionally, the hair bundle displacement produced by large force steps with a flexible fiber often possessed a secondary increase after the initial recoil. Further experiments are needed to determine whether this slower component of adaptation in the transducer current has a mechanical counterpart of reverse polarity in turtle hair cells.

\section{Mechanism of active hair bundle movements}

Our experiments do not identify the mechanism of the active bundle movements, although they do demonstrate a strong link to adaptation of the mechanotransducer channels. However, two questions seem particularly pertinent to defining the mechanism. Are the movements the cause of adaptation or a by-product of the changes in channel gating attributable to adaptation? Second, do the movements arise from a ciliary motor or from stiffness changes in a system under tension? The observation that the movements became less pronounced with larger stimuli, despite the prominence of adaptation (Fig. 3), would suggest that they are not directly responsible for adaptation. However, their speed and tem- 

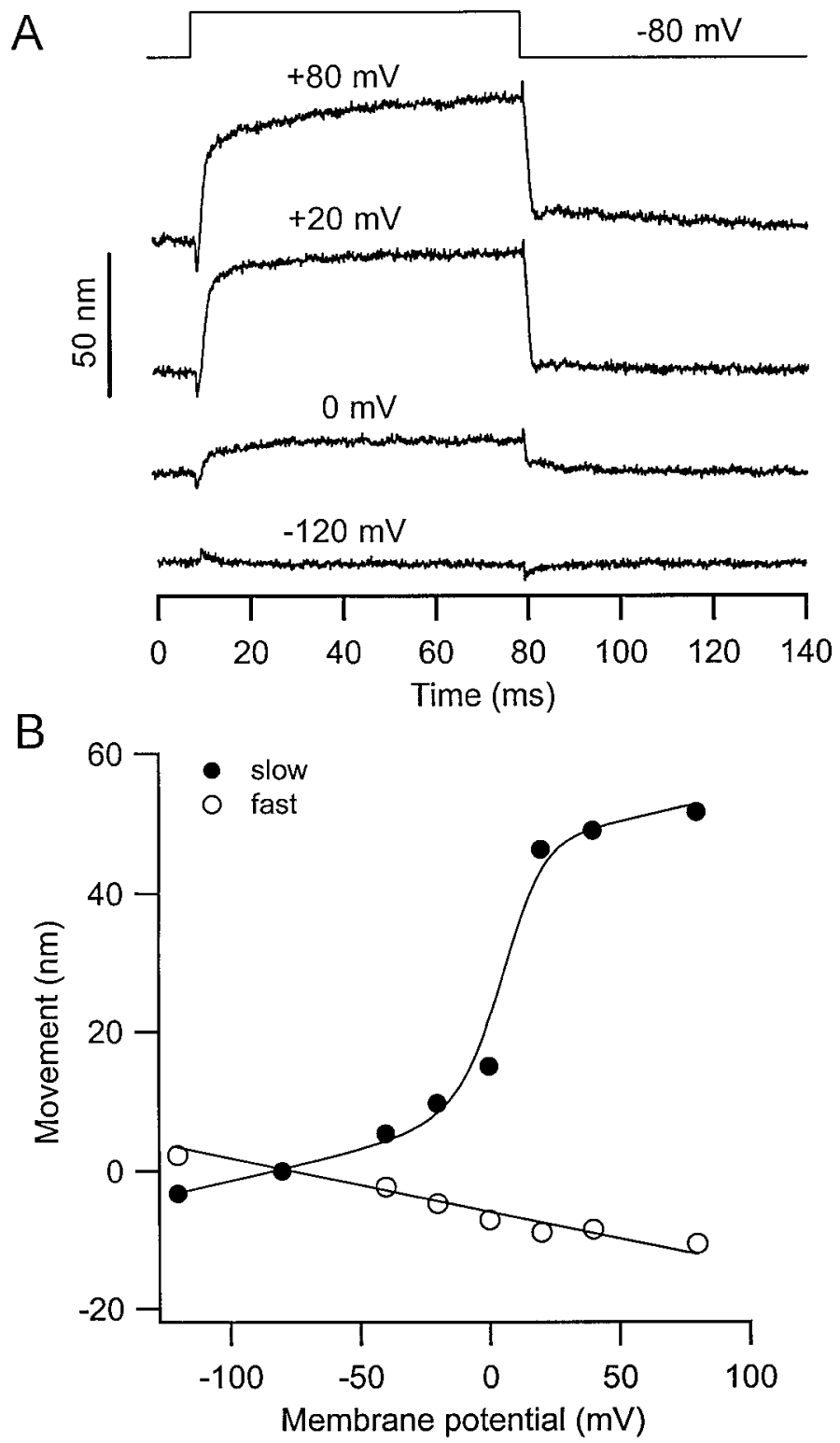

Figure 10. Voltage-dependence of hair bundle movements. A, Average movements for voltage steps from a holding potential of $-80 \mathrm{mV}$ to the membrane potential indicated above each trace. The responses consisted of a fast displacement, followed by a slower movement in the opposite direction. $B$, Plots of the amplitude of the fast and slow components of bundle motion against the membrane potential during the step. Note that the fast component (open circles) is proportional to membrane potential, but the larger slower component ( filled circles) develops only for depolarization positive to $0 \mathrm{mV}$. The curve through the filled circles was drawn by eye. Similar results were obtained in three other cells.

poral relationship to adaptation imply the operation of a very fast submillisecond force generator intimately associated with the channel. Indeed, the hair bundle movements might simply reflect the changes in hair bundle compliance secondary to molecular rearrangements of the channel during its gating cycle. This process, termed the "gating compliance," may be a significant fraction of the total compliance of the bundle (Howard and Hudspeth, 1988; Russell et al., 1992; van Netten and Khanna, 1994).

The gating compliance reflects the forces involved in the opening and closing of the mechanotransducer channels. It follows, therefore, that calcium interacting with the channel to alter its probability of opening must generate force and move the bundle. These
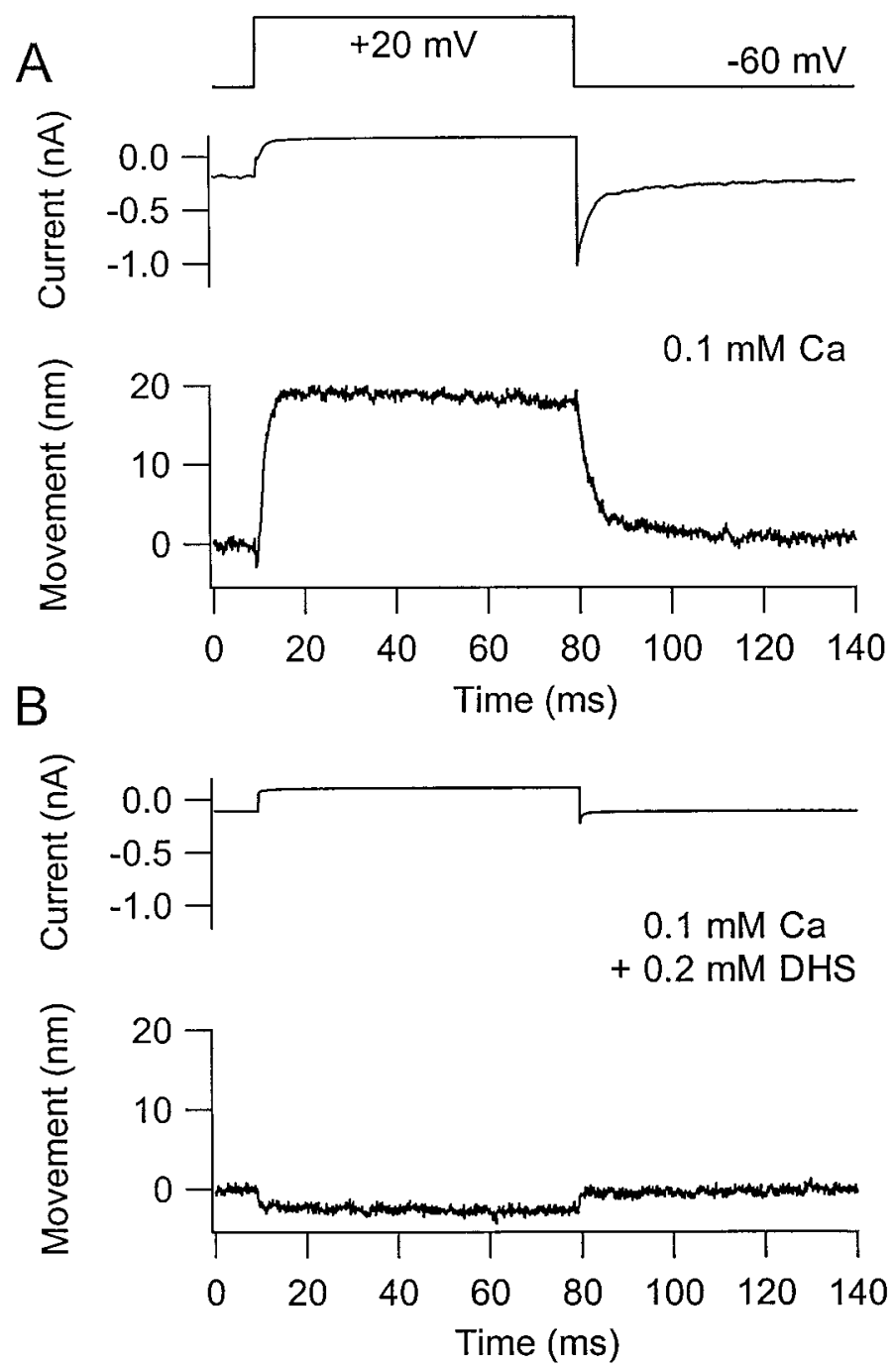

Figure 11. Effects of dihydrostreptomycin on the voltage-induced movements. $A$, Average current and bundle displacement for a depolarizing step from -60 to $+20 \mathrm{mV}$. The bundle motion consisted of a fast deflection, followed by a slower displacement in the opposite direction. $B$, DHS $(0.25$ $\mathrm{mM}$ ) abolished the slower component of movement, leaving a rectangular displacement similar to the time course of the voltage step. DHS also reduced the size of the current by blocking the transducer channels. Recordings were obtained in saline with reduced $(0.1 \mathrm{~mm})$ calcium to augment the potency of DHS

features of the mechanism are illustrated in Figure 12. First, it is assumed that fast adaptation results from calcium binding to the transducer channel complex to shift rapidly the activation curve of the channel (Fig. 12A) (Crawford et al., 1989, 1991). Under resting conditions (dashed curve), a small amount of calcium is bound, but increased calcium binding $(+C a)$ translates the activation curve along the displacement axis toward the kinocilium, and decreased calcium binding shifts it in the opposite direction. Second, gating of the transducer channel contributes significantly to the force-displacement relationship of the hair bundle (Fig. 12B) (Howard and Hudspeth, 1988). If the channels are all closed or all open, the plot is linear with slope reflecting the combined stiffness of the gating springs and the stereociliary pivots. However, bundle displacement opens the channels in a graded manner, generating a nonlinear force-displacement relationship. Under resting conditions, this relationship will have a form depicted by the dashed curve in Figure $12 B$ (Hudspeth, 1992; van Netten and Kros, 2000). Because of the effects of calcium on the transducer channel opening, calcium 

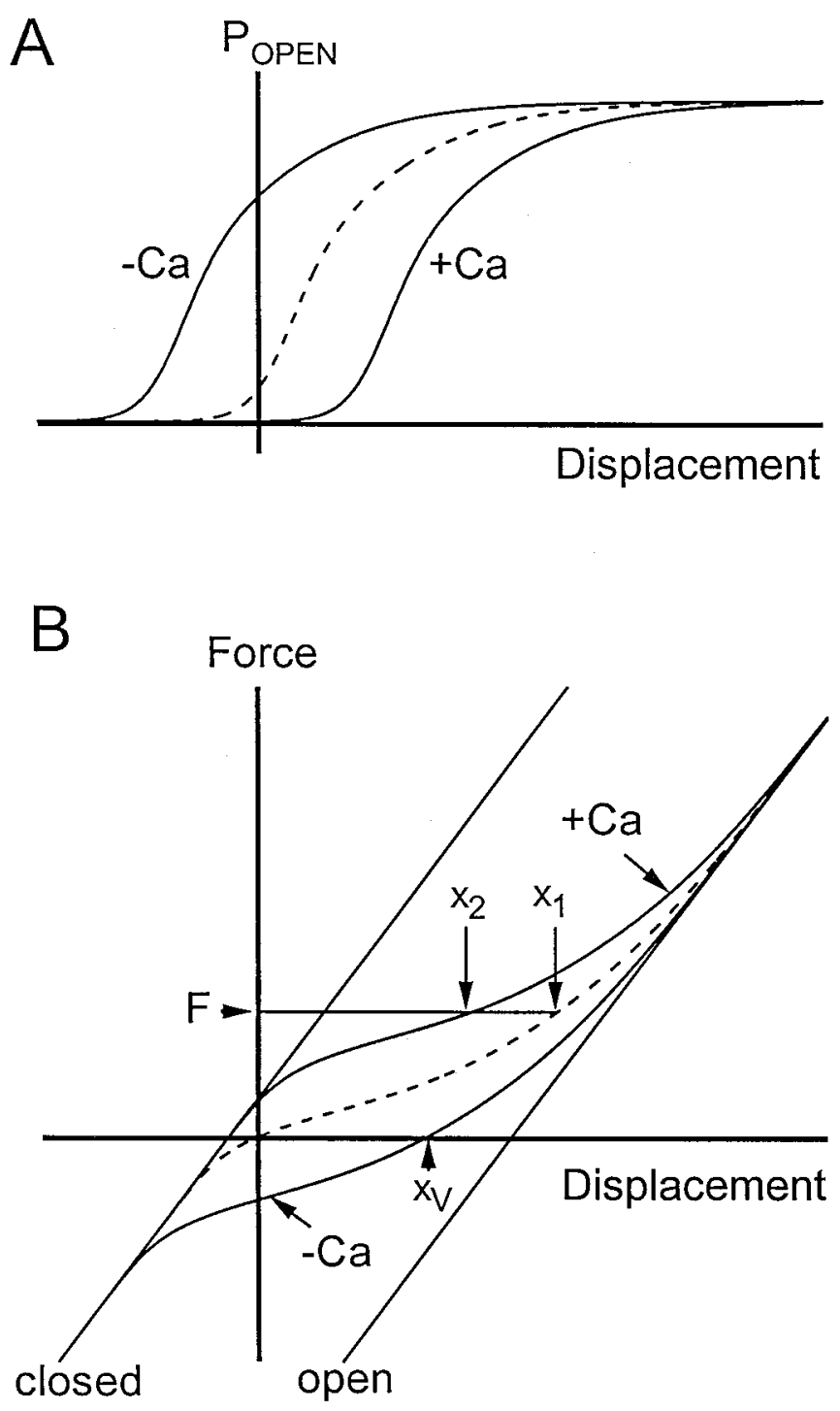

Figure 12. Schematic representation of the effects of intracellular calcium on hair bundle mechanics. $A$, Probability of opening of mechanoelectrical transducer channels with bundle displacement in different intracellular calcium concentrations. The relationship under resting conditions is represented by the dashed curve. With an increase in internal calcium, the channel complex binds more calcium $(+\mathrm{Ca})$, and the curve shifts to the right (toward the kinocilium). This shift is responsible for adaptation in the transducer current. With a decrease in intracellular calcium, as occurs during depolarization or reduction in extracellular calcium, the relationship shifts to the left $(-\mathrm{Ca})$. $\mathrm{B}$, Force-displacement relationship of the hair bundle depends on the state of the mechanoelectrical transducer channels. If all of the channels are fixed closed or open, the plot is linear with slope, reflecting the stiffness of the gating springs in parallel with the stereociliary pivots. However, the channels are free to open with bundle displacement, producing the nonlinear dashed curve under resting conditions. Because of the effects of calcium in $A$, an increase $(+C a)$ or decrease $(-C a)$ in calcium bound by the channel shifts the inflection in the force-displacement relationship. Thus, if the bundle is subject to a force step, $F$, the bundle initially moves along the dashed curve, producing a displacement $x_{1}$, but as calcium enters and binds to the channel $(+\mathrm{Ca})$, there is a reduction in displacement to $x_{2}$, reflecting channel closure. The figure also shows that reducing intracellular calcium (e.g., by depolarization) will cause a bundle movement $\left(\mathrm{x}_{V}\right)$ toward the kinocilium.

binding or unbinding is proposed to shift the inflection in the force-displacement relationship.

Figure $12 \mathrm{~B}$ can be used to illustrate the bundle motion induced by either a force step or a depolarization. If the bundle is subjected to a positive force step, $F$, it initially moves from the origin along the dashed curve, producing a peak displacement, $x_{1}$. The transducer channels open allowing influx of calcium, which binds to the channels, rapidly closing them and shifting the curve to $+C a$. Thus, the bundle recoils with a reduction in displacement to $x_{2}$, with a time course that parallels adaptation. Figure $12 B$ can also be used to illustrate the bundle movement caused by depolarization (van Netten and Kros, 2000). Assuming that depolarization beyond 0 $\mathrm{mV}$ reduces intracellular calcium, the force-displacement relationship will shift from rest (dashed curve) to one with less calcium bound $(-\mathrm{Ca})$. Therefore, in the absence of an applied force, the bundle will move from its resting position at the origin in the direction of the kinocilium to the point, $x_{V}$, at which the $-C a$ curve intersects the abscissa. The model of Figure 12, therefore, goes a long way toward explaining our results. However, further work is needed to establish the key assumption that bundle compliance can be significantly changed by manipulating intracellular calcium.

\section{REFERENCES}

Art JJ, Crawford AC, Fettiplace R (1986) A method for measuring cellular movements less than the wavelength of light. J Physiol (Lond) 371:18.

Assad JA, Corey DP (1992) An active motor model for adaptation by vertebrate hair cells. J Neurosci 12:3291-3309.

Assad JA, Hacohen N, Corey DP (1989) Voltage dependence of adaptation and active bundle movements in bullfrog saccular hair cells. Proc Natl Acad Sci USA 86:2918-2922.

Benser ME, Marquis RE, Hudspeth AJ (1996) Rapid, active hair bundle movements in hair cells from the bullfrog's sacculus. J Neurosci 16:5629-5643.

Corey DP, Hudspeth AJ (1979) Ionic basis of the receptor potential in a vertebrate hair cell. Nature 281:675-677.

Crawford AC, Fettiplace R (1985) The mechanical properties of ciliary bundles of turtle cochlear hair cells. J Physiol (Lond) 364:359-379.

Crawford AC, Evans MG, Fettiplace R (1989) Activation and adaptation of transducer currents in turtle hair cells. J Physiol (Lond) 419:405-434.

Crawford AC, Evans MG, Fettiplace R (1991) The actions of calcium on the mechano-electrical transducer currents in turtle hair cells. J Physiol (Lond) 434:369-398.

Denk W, Webb WW (1990) Optical measurement of picometer displacement of transparent microscopic objects. Appl Optics 29:2382-2391.

Denk W, Webb WW (1992) Forward and reverse transduction at the limit of sensitivity studied by correlating electrical and mechanical fluctuations in frog saccular hair cells. Hear Res 60:89-102.

Eatock RA (2000) Adaptation in hair cells. Annu Rev Neurosci 23:285-314.

Eatock RA, Corey DP, Hudspeth AJ (1987) Adaptation of mechanoelectrical transduction in hair cells of the bullfrog's sacculus. J Neurosci 7:2821-2836.

Fettiplace R, Fuchs PA (1999) Mechanisms of hair cell tuning. Annu Rev Physiol 61:809-834.

Flock A, Flock B, Murray E (1977) Studies on the sensory hairs of receptor cells in the inner ear. Acta Otolaryngol 83:85-91.

Hackney CM, Fettiplace R, Furness DN (1993) The functional morphology of stereociliary bundles on turtle cochlear hair cells. Hear Res 69:163-175.

Howard J, Ashmore JF (1986) Stiffness of sensory hair bundles in the sacculus of the frog. Hear Res 23:93-104.

Howard J, Hudspeth AJ (1987) Mechanical relaxation of the hair bundle mediates adaptation in mechanotransduction by the bullfrog's saccular hair cell. Proc Natl Acad Sci USA 84:3064-3068.

Howard J, Hudspeth AJ (1988) Compliance of the hair bundle associated with gating of mechanoelectrical transduction channels in the bullfrog's saccular hair cell. Neuron 1:189-199.

Hudspeth AJ (1982) Extracellular current flow and the site of transduction by vertebrate hair cells. J Neurosci 2:1-10.

Hudspeth AJ (1992) Hair-bundle mechanics and a model for mechanoelectrical transduction by hair cells. In: Sensory transduction (Corey DP, Roper SD, eds), pp 357-370. New York: Rockefeller UP.

Hudspeth AJ, Gillespie PG (1994) Pulling strings to tune transduction: adaptation by hair cells. Neuron 12:1-9.

Jaramillo F, Hudspeth AJ (1992) Localization of the hair cell's transduction channels at the hair bundle's top by iontophoretic application of a channel blocker. Neuron 7:409-420.

Martin P, Hudspeth AJ (1999) Active hair bundle movements can amplify a hair cell's response to oscillatory mechanical stimuli. Proc Natl Acad Sci USA 96:14306-14311.

Ohmori H (1985) Mechanotransduction currents in isolated vestibular hair cells of the chick. J Physiol (Lond) 359:189-217.

Pickles JO, Comis SD, Osborne MP (1984) Cross links between stereocilia in the guinea-pig organ of Corti, and their possible relation to sensory transduction. Hear Res 15:103-112. 
Ricci AJ, Fettiplace R (1997) The effects of calcium buffering and cyclic AMP on mechano-electrical transduction in turtle auditory hair cells. J Physiol (Lond) 501:111-124.

Ricci AJ, Fettiplace R (1998) Calcium permeation of the turtle hair cell's mechanotransducer channel and its relation to the composition of endolymph. J Physiol (Lond) 506:159-173.

Ricci AJ, Wu Y-C, Fettiplace R (1998) The endogenous calcium buffer and the time course of transducer adaptation in auditory hair cells J Neurosci 18:8261-8277.

Rüsch A, Thurm U (1990) Spontaneous and electrically induced movements of ampullary kinocilia and stereovilli. Hear Res 48:247-264.

Russell IJ, Kössl M, Richardson GP (1992) Nonlinear mechanical responses of mouse cochlear hair bundles. Proc R Soc Lond B Biol Sci 250:217-227.
Sigworth FJ (1983) Electronic design of the patch clamp. In: Singlechannel recording (Sakmann B, Neher E, eds) pp 3-35. New York: Plenum.

van Netten SM, Khanna SM (1994) Stiffness changes of the cupula associated with the mechanics of hair cells in the fish lateral line. Proc Natl Acad Sci USA 91:1549-1553.

van Netten SM, Kros CJ (2000) Gating energies and forces of the mammalian hair cell transducer channel and related hair bundle mechanics. Proc R Soc Lond B Biol Sci 267:1915-1923.

Weiss TF (1982) Bidirectional transduction in vertebrate hair cells. A mechanism for coupling electrical and mechanical processes. Hear Res 7:353-360.

Wu Y-C, Ricci AJ, Fettiplace R (1999) Two components of transducer adaptation in auditory hair cells. J Neurophysiol 82:2171-2181. 